\title{
Dewetting of thin polymer films near the glass transition
}

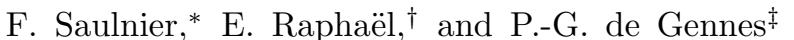 \\ Laboratoire de Physique de la Matière Condensée, CNRS UMR 7125, Collège de France \\ 11, place Marcelin Berthelot, 75231 Paris Cedex 05, France.
}

(Dated: 28th of january, 2002)

\begin{abstract}
Dewetting of ultra-thin polymer films near the glass transition exhibits unexpected front morphologies [G. Reiter, Phys. Rev. Lett., 87, 186101 (2001)]. We present here the first theoretical attempt to understand these features, focusing on the shear-thinning behaviour of these films. We analyse the profile of the dewetting film, and characterize the time evolution of the dry region radius, $R_{d}(t)$, and of the rim height, $h_{m}(t)$. After a transient time depending on the initial thickness, $h_{m}(t)$ grows like $\sqrt{t}$ while $R_{d}(t)$ increases like $\exp (\sqrt{t})$. Different regimes of growth are expected, depending on the initial film thickness and experimental time range.
\end{abstract}

PACS numbers: 68.60.-p, 68.15.+e, 68.55.-a, 83.10.-y

Thin liquids films are ubiquitous entities in science and technology. In engineering, for instance, they serve to protect surfaces, and applications arise in paints, adhesives and membranes [1]. Thin liquids films display a variety of interesting dynamics phenomena and have therefore been the focus of many experimental and theoretical studies [2]. Nearly half a century ago, Taylor [3] and Culick [4] analyzed the growth of a circular hole in a thin liquid sheet [5]. By balancing surface tension forces against inertia, they found that the rim of liquid at the edge of the films retracts at a constant velocity, a prediction first checked experimentally by Mc Entee and Mysels [6]. The precise shape of the rim was later analysed by Keller et al. [7, 8] who showed that it is a cylindrical cap expanding in time like $\sqrt{t}$. Recent experiments by Debrégeas and collaborators [9] on thin suspended films of very viscous liquids revealed unexpected features: (a) First, the retraction velocity grows exponentially with time (with a characteristic time scale $\tau_{i}=h_{i} \eta /|S|$, where $h_{i}, \eta$ and $S$ are respectively the initial film thickness, the viscosity and the spreading coefficient [10]), (b) second, the liquid is not collected into a rim and the film remains flat through the retraction. According to these authors, the uniform thickening of the retracting film was a consequence of its viscoelasticity, which permits an elastic propagation into the film of the surface tension forces acting on the edge. Solving numerically the Navier-Stokes equations for long wavelength modulations of the film, Brenner and Gueyffier [11] showed, however, that the absence of rim can also result from a purely viscous effect.

Very recently [12], Reiter studied the dewetting of ultrathin (i.e. thinner than the coil size), almost glassy polystyrene (PS) films deposited onto silicon wafers coated with a polydimethylsiloxane (PDMS) monolayer. He found that a highly asymmetric rim, with an extremely steep side towards the interior of the hole and

\footnotetext{
*Electronic address: florent.saulnier@college-de-france.fr

${ }^{\dagger}$ Electronic address: lie.raphael@college-de-france.fr

${ }^{\ddagger}$ Electronic address:
}

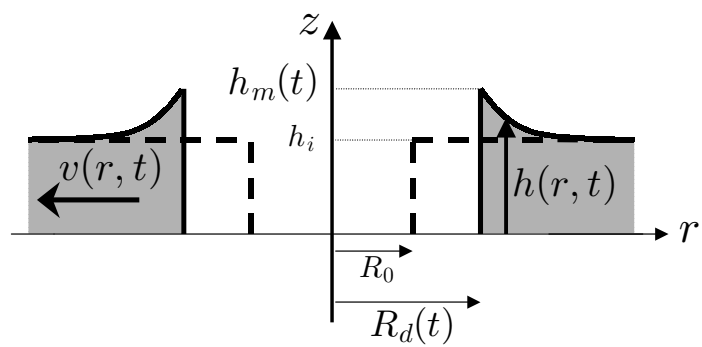

FIG. 1: Film geometry: $h(r, t)$ is the profile of the film, $h_{m}(t)$ is the height of the rim, and $R_{d}(t)$ is the radius of the dry zone. The initial $(t=0)$ step-like profile is represented by the dashed line. $v(r, t)$ is the radial, axisymmetric flow field [14].

a much slower decay on the rear side, builds up progressively 13]. In this Letter we present the first theoretical attempt to understand these new features. Figure 1 shows the film geometry.

In order to characterise the rheologic properties of the film, we introduce the stress tensor $\sigma_{i j}=-p \delta_{i j}+\sigma_{i j}^{m}$, where $p$ is the pressure and $\sigma_{i j}^{m}$ represents the effects of internal friction. We relate $\sigma_{i j}^{m}$ to the strain rate tensor $\dot{\gamma}_{i j}$ by a constitutive law of the form:

$$
\sigma^{m}(\dot{\gamma})=\sigma_{0} \Phi(\dot{\gamma} \tau)
$$

where $\sigma_{0}$ and $\tau$ are material constants, and $\Phi$ is a generic function. For a purely viscous liquid, the function $\Phi$ is linear, whereas for polymers, just above $T_{g}$, it can be shown within the framework of the free-volume model 115. that $\sigma^{m}$ is expected to vary logarithmically with $\dot{\gamma}$ as [16]:

$$
\Phi(\dot{\gamma} \tau)=\ln (1+\dot{\gamma} \tau)
$$

At low strain-rates $(\dot{\gamma} \tau<1)$, this law displays a viscous-type behaviour ( $\sigma^{m} \approx \eta_{0} \dot{\gamma}$, with a zero-shear viscosity $\eta_{0}=\sigma_{0} \tau$ ), while for large values of $\dot{\gamma} \tau, \sigma^{m}$ reaches an almost constant value (shear-thinning behaviour). 


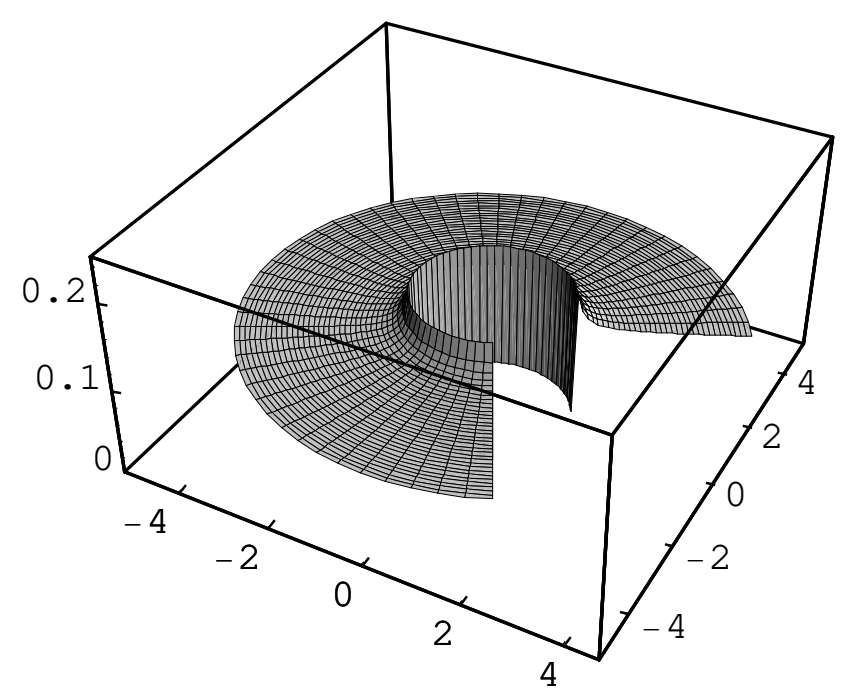

FIG. 2: Film profile for $h_{i}=0.1 h^{*}$ and $t=10^{-2} \tau$. For clarity reasons, a sectional view is displayed on a quarter of circle.

Assuming the fluid to be incompressible, mass conservation leads to:

$$
\frac{1}{h(r, t)}\left(\frac{\partial h(r, t)}{\partial t}+\frac{r \beta(r, t)}{\tau} \frac{\partial h(r, t)}{\partial r}\right)=\frac{\alpha(r, t)-\beta(r, t)}{\tau}
$$

Equation 3 involves two unknown functions $\alpha(r, t)$ and $\beta(r, t)$, that are positive, dimensionless forms of the strain-rate components $\dot{\gamma}_{r r}$ and $\dot{\gamma}_{\phi \phi}$ :

$$
\left\{\begin{array}{l}
\alpha=-\tau \dot{\gamma}_{r r}=-\tau \frac{\partial v}{\partial r} \\
\beta=\tau \dot{\gamma}_{\phi \phi}=\tau \frac{v}{r}
\end{array}\right.
$$

We thus need two additional equations to determine $h(r, t)$. First, note that the following partial differential equation can be directly derived from Eq. 4 :

$$
\frac{\partial \beta}{\partial r}=-\frac{\beta+\alpha}{r}
$$

Neglecting the inertial term, conservation of momentum (projected on the radial direction [17) leads to:

$$
\frac{\partial \sigma_{r r}}{\partial r}+\frac{\sigma_{r r}-\sigma_{\phi \phi}}{r}=0
$$

It can be shown that Eqs. 5 and 6 , along with the freesurface boundary condition (i.e. $\sigma_{z z}=0$ at the contact with ambient atmosphere [18]), allow one to express the strain rate $\alpha$ as a function of $\beta$ only : $\alpha=F(\beta)$. Substitution of the constitutive law 1 in Eq.6 then leads to the following differential equation for $F$ :

$$
\frac{\mathrm{d}[\Phi(F(\beta))-\Phi(F(\beta)-\beta)]}{\mathrm{d} \beta}=\frac{\Phi(F(\beta))-\Phi(\beta)}{F(\beta)+\beta}
$$

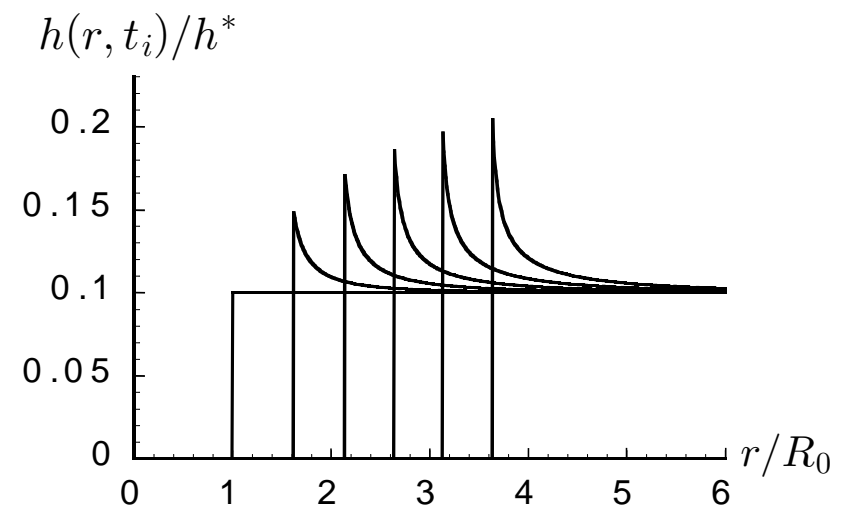

FIG. 3: Evolution of the film profile $h(r, t)$ for $h_{i}=0.1 h^{*}$ and $t$ ranging from $t=0$ to $t=5 \cdot 10^{-2} \tau$ with a time step of $10^{-2} \tau$.

which has to be solved along with the condition $F(0)=$ 0 (far away from the perturbed central region, the strain rates must decrease to zero).

In order to solve Eq.3, we should supply it with initial and boundary conditions. Our initial profile is assumed to be uniform, with a thickness $h_{i}$, except in a bored region ranging from $r=0$ to $r=R_{0}$ (as shown in fig. (1). $R_{0}$ is our characteristic radial length used thereafter to make $r$ dimensionless:

$$
h(r, t=0)= \begin{cases}h_{i} & \text { if } r \geq R_{0}=1 \\ 0 & \text { otherwise }\end{cases}
$$

We do not consider here the origin of the initial dewetting process: experimentally, it is found that a thick PS film on a silicon substrate is metastable and dewets via nucleation and growth of dry patches [19], while thinner films $\left(h_{i}<100 \mathrm{~nm}\right)$ are unstable and dewet by spinodal decomposition 20]. In his latest experiments on ultrathin films [12], Reiter characterized the early stage of the dewetting process by the formation and coalescence of little holes, with the displaced material uniformly distributed between the holes, without visible rims. Our initial time $t=0$ might correspond to the end of this preliminary process.

Equation 3 applies outside the dewetted region $\left(R_{d}(t) \leq r<\infty\right)$. At the edge of the $\operatorname{rim}\left(r=R_{d}(t)\right)$, the rim height, $h_{m}(t)$, can be determined by taking into account capillary forces. The applied force on the rim, pushing the film away the dry area, must be balanced by the internal radial stress: $|S|=\left|\sigma_{r r}\right| h_{m}(t)$. Assuming the lateral extension of the film to be large enough, the film thickness must reach $h_{i}$ far from the dry region: $\lim _{r \rightarrow \infty} h(r, t)=h_{i}(\forall t)$. The complete resolution of our set of equations 3 5 5 - can be achieved using a method of characteristics 15]. Thereafter, all thicknesses will be made dimensionless by normalizing with a characteristic length $h^{*} \equiv|S| / \sigma_{0}$ (for Reiter's experimental conditions, we estimate $h^{*}$ to be of the order of $500 \AA$ ).

Note first that for a purely viscous liquid $(\Phi(\dot{\gamma} \tau)=\dot{\gamma} \tau)$, 


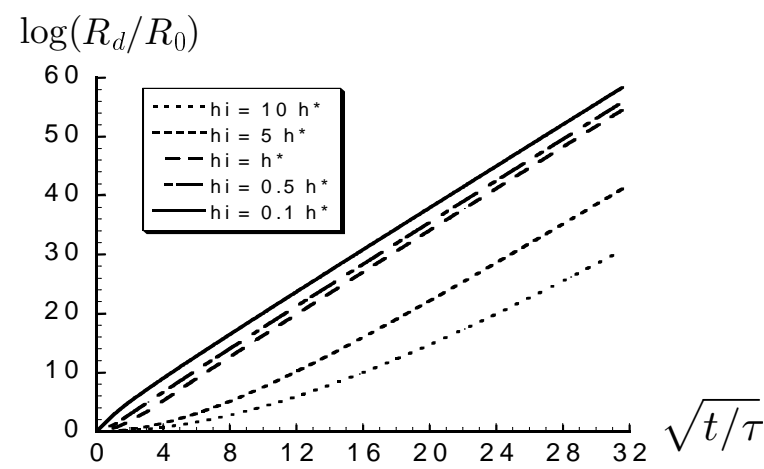

FIG. 4: Logarithmic plot of the dry zone radius $R_{d}$ versus $\sqrt{t / \tau}$, for $h_{i}=0.1,0.5,1,5$ and $10 h^{*}$. Note the linear behaviour of $\log \left[R_{d}(t) / R_{0}\right]$ with $\sqrt{t / \tau}$ after a transient time.

our model leads to a constant and uniform thickness for the film, with an exponential growth of the dry radius:

$$
R_{d}(t)=R_{0} e^{\frac{|S| t}{\sigma_{0} \tau h_{i}}}=R_{0} e^{\frac{t}{\tau_{i}}}
$$

This is in complete agreement with the experimental results of Debrégeas et al. for the dewetting of suspended polymer films [9] and supported films [21].

If we take into account the shear-thinning behavior of the polymer film $\left(\sigma^{m}(\dot{\gamma})=\sigma_{0} \ln (1+\dot{\gamma} \tau)\right)$ the dewetting front is quite different. An example of profile obtained for $h_{i}=0.1 h^{*}$ is shown in Fig.2. This profile is characterized by a highly asymmetric shape for the rim. There is a striking similarity between such a profile and those observed by Reiter for ultra-thin films [12]. On fig. 3 we present the time evolution of this profile (for $t$ ranging from $t=0$ to $t=5 \cdot 10^{-2} \tau$, with a time step of $10^{-2} \tau$ ). The velocity field $v(r, t)$ is found to be almost proportional to $1 / r$ at any time for thick profiles $\left(h_{i} \gg h^{*}\right)$, similar to the long range radial plug flow observed by Debrégeas et al. [9]. We now focus on two natural parameters characterizing the film profile: the dry radius $R_{d}(t)$ and rim height $h_{m}(t)$.

In order to discuss the time dependence of the rim height, we need to compare $h_{i}$ with the characteristic thickness $h^{*}$ (in Fig. 5 we present our results for different initial thicknesses). For very thin profiles $\left(h_{i} \ll h^{*}\right)$, the increase of rim height is initially very fast, whereas for thicker films $\left(h_{i} \gg h^{*}\right), h_{m}(t)$ is nearly constant during a long period of time, before growing faster. For the sake of conciseness, we will mainly focus on the case of thick films, for which simple scaling laws can be analytically obtained. As shown in Fig. 5 , for $h_{i}=100 h^{*}$ two different time regimes can be distinguished: at the beginning of hole formation, $h_{m}$ is nearly constant and equals its initial value $h_{i}$. We have checked numerically (cf. Fig.5 b) that the anticipated analytical behaviour

$$
h_{m}(t) \underset{t \gg t_{0}}{\sim} \frac{h^{*}}{2} \sqrt{t / \tau}
$$

is obeyed after a crossover time $t_{0} \approx \tau\left(\frac{h_{i}}{h^{*}}\right)^{2}$

In Fig. 1 , we present the time evolution of $R_{d}$ for different initial thicknesses $h_{i}$. It is physically understandable that $R_{d}$, at a given time $t$, is larger for thinner films : for a given applied force $|S|$ per unit length, the thicker the film, the more the material to be displaced and the lower the dewetting velocity. Fig. 1 shows that the following anticipated analytical behaviour is obeyed for $t \gg t_{0}$ :

$$
R_{d}(t) \underset{t \gg t_{0}}{\sim} R_{\infty} e^{4 \sqrt{t / \tau}}
$$

Note that the coefficient $R_{\infty}$ strongly depends on $h_{i}$. During the early stage $\left(t \ll t_{0}\right)$, the strain rates at the rim are constant and small, as a consequence of the constant large thickness. In this case, the rheological law of the film is viscous-type and the dry radius increases exponentially, as for the case previously discussed (see Eq.9). The range of thicknesses experimentally studied by Debrégeas et al. (5 to $250 \mu \mathrm{m}$ ) covers the domain $h_{i} \gg h^{*}$. As the corresponding crossover time is very large $\left(t_{0} \gg \tau\right)$, these experiments only covered mainly the first regime $t \ll t_{0}$ (this criterion $h_{i} \gg h^{*}$ is in fact a basic hypothesis of their "soft balloon" model [22]). The crossover to the $\exp (\sqrt{t})$ regime is a consequence of the non-linearity of rheological law 2 .

The case $h_{i} \ll h^{*}$ will not be fully discussed here (cf. [15). Briefly, we can note on Fig. 5 that a sharp rim grows very quickly (with an initial speed $\dot{h}_{m} \approx h_{i} \exp h^{*} / 2 h_{i}$ ) at short times. In this regime, $h_{m}(t)$ is well described by the integral equation:

$$
\int_{\frac{h^{*}}{2 h_{m}(t)}}^{\frac{h^{*}}{2 h_{i}}} \frac{e^{-x}}{x} \mathrm{~d} x \underset{t \ll \tau}{\approx} \frac{t}{\tau}
$$

Later on, $h_{m}$ and $R_{d}$ reach the same laws of growth as in the case of thick films (Eqs.10 and 11).

In conclusion, our model accounts well for the exponential growth and the absence of rim characteristics of the dewetting regime observed by Debrégeas et al. for viscous polymer films. Taking into account the shearthinning behaviour of the polymers near $T_{g}$ enables us to see the modifications induced by this particular rheology on the film morphology [23]. It appears from our results that the early stages of dewetting for a shear-thinning polymer film are mainly determined by the ratio of its initial thickness $h_{i}$ to a characteristic scale $h^{*}$ (related to the driving force of the process, $S$, and the rheological response of the material, characterized by $\sigma_{0}$ ). In all cases, after a transient time depending on $h_{i}$, the rim height grows proportionally to $\sqrt{t / \tau}$ while the dry radius expands like $\exp (4 \sqrt{t / \tau})$. The film profile exhibits a sharp, asymmetric rim similar to the one observed by Reiter in his latest experiments with ultrathin PS films. Work is now in progress to incorporate in our model the Laplace pressure (see [18 ) which might lead to oscillatory dewetting fronts 11, 13], and to evaluate the possible effects 

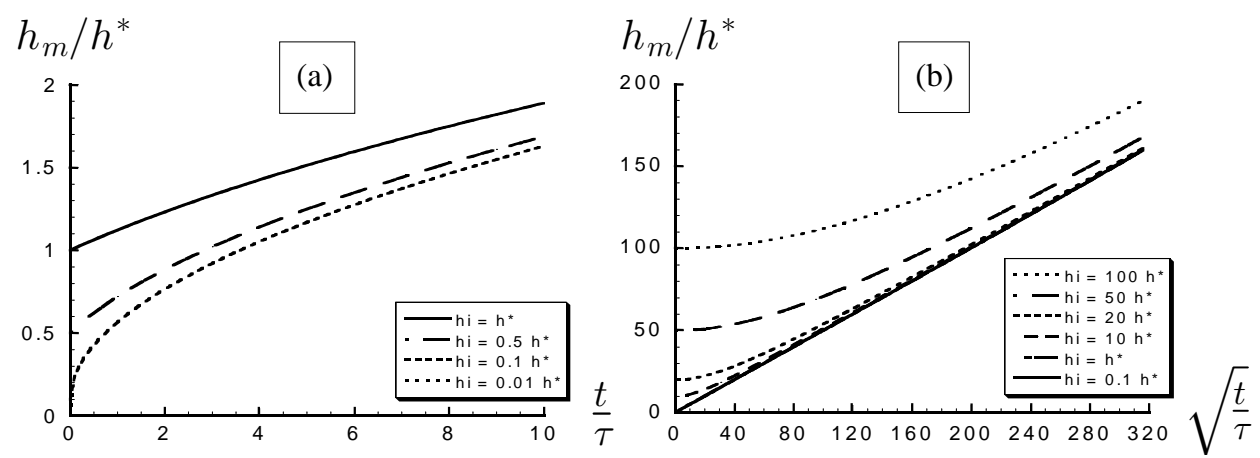

FIG. 5: Time evolution of the rim height $h_{m}$ : (a) Plot of $h_{m}$ versus $t / \tau$, for $h_{i}=0.01,0.1,0.5$ and $1 h^{*}$. Note that the curves corresponding to $h_{i}=0.1$ and $0.01 h^{*}$ cannot be distinguished within the graph scale. (b) Plot versus $\sqrt{t / \tau}$ to show the linear behaviour of $h_{m}$ with $\sqrt{t / \tau}$ after the transient time $t_{0}$. For a thick film $\left(h_{i} \gg h^{*}\right)$, two regimes can be distinguished. For $t<t_{0} \sim \tau\left(\frac{h_{i}}{h^{*}}\right)^{2}, h_{m}$ is constant and $R_{d}(t)$ increases exponentially with time. For $t>t_{0}, h_{m}$ growths linearly with $\sqrt{t / \tau}$ and $R_{d}(t)$ expands like $\exp \sqrt{t / \tau}$.

of chain entanglements. In the future, we aim to study the dewetting of thin polymer films below $T_{g}$, where the existence of a yield stress in the rheological response of the material may lead to new dewetting morphologies.

We wish to acknowledge fruitful discussions with A. Aradian, G. Debrégeas, and G. Reiter.
[1] A. Oron, S. H. Davis, and S. G. Bankoff, Rev. Mod. Phys. 69, 931 (1997)

[2] See e.g., G. Reiter, Science 282, 888 (1998); S. Herminghaus et al., Science 282, 916 (1998)

[3] G. I. Taylor, Proc. R. Soc. London, Ser. A. 253, 313 (1959)

[4] F. E. C. Culick, J. Appl. Phys. 31, 1128 (1960)

[5] For earlier work on the subject, see: A. Dupré, Ann. Chim. Phys. 11, 194 (1867)

[6] W. R. Mc Entee, and K. J. Mysels, J. Phys. Chem. 73, 3018/3028 (1969)

[7] J. B. Keller, A. King, and L. Ting, Phys. Fluids 7, 226 (1995)

[8] For molecular simulations on the fluids dynamics of the moving rim, see: J. Koplik, and J. R. Banavar, Phys. Rev. Lett. 84, 4401 (2000)

[9] G. Debrégeas, P. Martin, and F. Brochard-Wyart, Phys. Rev. Lett. 75, 3886 (1995);

G. Debrégeas, P.-G. de Gennes, and F. Brochard-Wyart, Science 279, 1704 (1998)

[10] The driving force of the dewetting process is a difference of interfacial tensions: $-S=\gamma_{S L}+\gamma_{L O}-\gamma_{S O}>0$ (where SL corresponds to the solid/liquid interface, SO to solid/air interface, etc...).

[11] M. P. Brenner and D. Gueyffier, Phys. Fluids 11, 737 (1999)

[12] G. Reiter, Phys. Rev. Lett. 87, 186101 (2001)

[13] For somewhat related studies, see: R. Seemann, S. Herminghaus, and K. Jacobs, Phys. Rev. Lett. 87, 196101 (2001)

[14] On a non-wettable, smooth and passive solid substrate like the PDMS-coated silicon wafer used by Reiter, this plug-flow description is valid as long as $h_{i} \ll b$ where $b$ is the hydrodynamic extrapolation length (cf. P.-G. de Gennes, C. R. Acad. Sci. 288B, 219 (1979)).

[15] F. Saulnier, E. Raphaël, and P.-G. de Gennes (in prepa- ration). In order to deal with a minimum number of parameters, we assume that $\sigma^{m}(\dot{\gamma})$ is an odd function $\left(\sigma^{m}(\dot{\gamma})=-\sigma_{0} \ln (1+|\dot{\gamma}| \tau)\right.$ if $\left.\dot{\gamma}<0\right)$.

[16] This Wagner-type constitutive relation can also be obtained from a purely rheological approach, based on the Cox-Merz rule and Eyring's expression for the nonlinear shear viscosity : see J. C. Dyre, Rheol. Acta. 29, 145 (1990)

[17] R. M. Christensen, Theory of viscoelasticity, an introduction (Academic Press, 2nd edition, 1982), p.53

[18] In order to keep the model analytically tractable, we here neglect the pressure discontinuity arising from the interface curvature. Further work will be needed to reassess the range of validity of this assumption.

[19] C. Redon, F. Brochard-Wyart, and F. Rondelez, Phys. Rev. Lett. 66, 715 (1991)

[20] G. Reiter, Phys. Rev. Lett. 68, 75 (1992)

[21] F. Brochard-Wyart et al., Macromolecules 30, 1211 (1997). This exponential behaviour is observed during the initial stage of process, as long as the viscous friction on the liquid/substrate interface remains negligible compared with the dissipation in the plug flow of removing fluid.

[22] It is clear that this exponential law cannot be valid for too thin films. Writing $\eta=E \tau_{r e p}$ (where $E$ is an elastic modulus, and $\tau_{r e p}$ is the reptation time of polymers, the relaxation time of the material), one obtains $\tau_{i}=\tau_{r e p} h / h_{0}$ with $h_{0} \equiv|S| / E$, which leads to a characteristic time $\tau$ smaller than $\tau_{r e p}$ if $h_{i}<h_{0}$ : the relaxation would be too fast.

[23] See the related experimental work of K. Dalnoki-Veress et al., Phys. Rev. E, 59, 2153 (1999), where the formation and growth in freely standing PS films is analyzed in terms of shear-thinning properties. 